\section{The Languages of Oncology}

A presenter at a recent conference had a wonderful slide, a photograph of a street sign that said, "Dog owners: You are required to clean up after your dog. Dogs: Woof, ruff, grrrr, good dog!"

The point of the slide, the presenter noted, is that it highlights the need to use the right language for the audience. This is an important philosophy in oncology, and it is especially important at NCCN. We develop clinical content in a number of formats, each for a different primary audience. NCCN Clinical Practice Guidelines in Oncology are targeted to the clinical professional and written in medical terminology. Additionally, a number of the guidelines are being translated into Chinese, Japanese, Spanish, and other languages to serve oncologists in other parts of the world.

$\mathrm{NCCN}$ is also developing a set of standard orders templates that provide explicit information about drug regimens named in the guidelines. These are drafted by oncology pharmacists and reviewed by both physicians and nurses to ensure that the language used in them is both accurate and understandable to those audiences.

But clinicians are not our only audience. A number of the guidelines are "translated" into more patient-friendly language in a cooperative effort with American Cancer Society to help more medically sophisticated patients (the literacy rate is quite high) understand the technical clinical decisions that drive their care. Guidelines are also translated into the NCCN Drugs and Biologics Compendium, which includes an alphabetical listing of all agents discussed in our guidelines to guide evaluation of their use by payors and clinicians' business managers.

Recently, a new project has involved NCCN staff in yet another language, that of databases, data models, and use cases. This is the language we needed to learn in working with a software integration firm to develop a new guidelines user system. This system will also have several audiences and must support both ease of use for the guidelines users on the web and maintenance of guidelines by NCCN staff.

In addition to introducing NCCN to this new language, the guidelines user system reminds us of the true purpose of language-communication. By placing the guidelines recommendations in a database that includes positional information about what comes before and after each recommendation, as well as its Category of Evidence and Consensus and the evidence on which it is made, we believe we can communicate more of the richness of the guidelines process to users. This new database-driven system should also allow the NCCN guidelines to become "multilingual"-able to communicate with other systems and therefore to be incorporated more easily into electronic medical records, computerized order entry systems, decision-assist tools, and quality evaluation tools.

The opportunities to improve patient care grow as we find new ways to craft language appropriate for our various audiences of professionals and patients. Focusing on pancreatic cancer and fatigue, this issue of JNCCN, like that multi-audience sign, also highlights the many languages of oncology - the languages of genetics inherent in the discussion of prognostic markers, the language of chemistry involved in developing new agents, the language needed to evaluate fatigue in reproducible ways, and the language of patients trying to understand what informed consent documents mean for them.

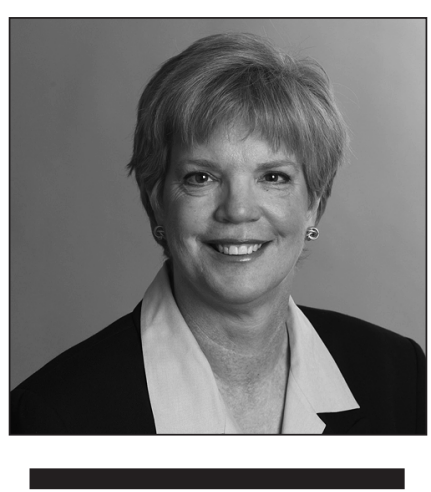

Joan S. McClure, MS

Joan S. McClure, MS, is Senior Vice President of Clinical Information and Publications for the NCCN. She is responsible for the NCCN Clinical Practice Guidelines in Oncology; associated Guidelines for Patients in both English and Spanish; the NCCN Drugs \& Biologics Compendium; NCCN Information Technology; and JNCCN; and also serves as an Associate Editor for JNCCN. Ms. McClure previously managed national oncology information programs on contract with the U.S. National Cancer Institute (NCl). She directed investigator and patient recruitment efforts in oncology for a multinational contract research organization, where she also managed efforts to identify and develop standards for data for submission to regulatory authorities in the United States, Europe, and Japan.

The ideas and viewpoints expressed in this editorial are those of the author and do not necessarily represent any policy, position, or program of the NCCN. 